[0212-7199 (2003) 20: 11; pp 579-581] Copyright (C) 2003 ARAN EDICIONES, S.L.

AN. MED. INTERNA (Madrid) Vol. 20, N. ${ }^{\circ} 11$, pp. $579-581,2003$

\title{
Hepatitis isquémica en pacientes con insuficiencia cardiaca
}

\author{
J. MUÑOZ-RODRÍGUEZ, J. M. TRICAS LERIS, V. ANDREU SOLSONA', \\ J. VILASECA BELLSOLÀ
}

Servicio de Medicina Interna $y^{1}$ Unidad de Digestivo. Hospital de Mollet. Mollet del Vallès. Barcelona

ISCHEMIC HEPATITIS IN PATIENTS WITH HEART FAILURE

\section{RESUMEN}

La hepatitis isquémica o hipóxica es una entidad clínica caracterizada por una importante elevación de las transaminasas de forma aguda y reversible que ocurre en aquellas situaciones que pueden ocasionar una reducción del flujo sanguíneo hepático, especialmente la insuficiencia cardiaca, siempre que se hayan descartado previamente otras causas potencialmente responsables de una hepatitis aguda, especialmente víricas y farmacológicas. Histológicamente se caracteriza por una necrosis centrolobulillar. Presentamos los 3 casos ingresados en un servicio de medicina interna durante un periodo de un año. Su incidencia fue del $2,7 \%$ entre todos los pacientes ingresados durante el mismo periodo con el diagnóstico de insuficiencia cardiaca.

PALABRAS CLAVE: Hepatitis isquémica. Insuficiencia cardiaca. Pronóstico.

\begin{abstract}
Ischemic hepatitis is characterized by a marked and reversible elevation in either the serum alanine or aspartate aminotransferase level in a appropriate clinical setting that could lead to a reduction in hepatic blood flow, mainly in patients with heart failure. To establish the diagnosis other causes of hepatitis, such as virus and drugs, must be previously excluded. Centrilobular necrosis is the main histologic feature. In the present study we describe the three cases of ischemic hepatitis seen in our medical service during a period of one year. Its prevalence was $2.7 \%$ among all patients with heart failure admitted in our centre during the same period.
\end{abstract}

KEY WORDS: Ischemic hepatitis. Heart failure. Prognosis.

Muñoz-Rodríguez FJ, Tricas Leris JM, Andreu Solsona V, Vilaseca Bellsolà J. Hepatitis isquémica en pacientes con insuficiencia cardiaca. An Med Interna (Madrid) 2003; 20: 579-581.

\section{INTRODUCCIÓN}

La hepatitis isquémica o hipóxica se caracteriza por una importante elevación de las transaminasas de forma aguda y reversible que, en ausencia de otras causas potencialmente responsables de una hepatitis aguda, sucede en un contexto clínico responsable de una reducción del flujo sanguíneo hepático (1). Histológicamente se caracteriza por una necrosis centrolobulillar $(2,3)$, aunque actualmente se acepta que no es necesaria la confirmación histológica para establecer su diagnóstico. Presentamos 3 casos de hepatitis isquémica ingresados en nuestro centro hospitalario en un periodo de 1 año.

\section{CASOS APORTADOS}

Caso 1. Paciente mujer de 75 años de edad con antecedentes de hipertensión arterial, fibrilación auricular crónica y cardiopatía isquémica, que ingresó por un cuadro clínico de 3 días de evolución caracterizado por disnea de reposo, ortopnea y palpitaciones. La exploración física puso de manifiesto la existencia de crepitantes pulmonares bilaterales, taquiarritmia, ingurgitación yugular y edemas en las extremidades inferiores. En la radiografía torácica se observaron signos compatibles con edema pulmonar y en la gasometría arterial basal, una insuficiencia respiratoria aguda hipocápnica. El ECG reveló una fibrilación auricular con una respuesta ventricular media de 150 lx'. En la analítica de ingreso destacaba una intensa citolisis hepática (GOT 2280 U/L, GPT 2600 U/L y bilirrubina total $4,2 \mathrm{mg} / \mathrm{dl}$ ). Las serologías para los virus de la hepatitis A, B y C fueron negativas. En la ecografía abdominal se observó un hígado de estasis con dilatación de las venas suprahepáticas. Se inició tratamiento con diuréticos, digitálicos y amiodarona. La paciente presentó una evolución favorable con una mejoría progresiva de los signos de fallo cardiaco y normalización de la biología hepática.

Caso 2. Paciente mujer de 79 años que ingresó por una infección respiratoria complicada con hiperreactividad bronquial e insuficiencia respiratoria aguda hipercápnica. Posteriormente presentó un episodio de fibrilación auricular con respuesta ventricular rápida e hipo-

Trabajo aceptado: 29 de abril de 2003

Correspondencia: Fco. José Muñoz-Rodríguez. Servicio de Medicina Interna. Hospital de Mollet. C/ Sant Llorenç 39-41. 08100 Mollet del Vallès. Barcelona. e-mail: 28587fmr@comb.es 
tensión arterial. Se controló la frecuencia cardiaca con digitálicos y se normalizó la tensión arterial. En la analítica de ingreso se observó una importante citolisis (GOT 2080 U/L, GPT 3600 U/L, bilirrubina 1,65 mg/dl, Quick 41\%). Las serologías para los virus de la hepatitis A, B y C fueron negativas. En una ecocardiografía se observó la existencia de hipertensión pulmonar moderada con dilatación del ventrículo derecho, todo ello compatible con cor pulmonale. Tras la mejoría clínica de la paciente, se produjo una rápida normalización de las transaminasas.

Caso 3. Paciente mujer de 68 años de edad que refería antecedentes patológicos de asbestosis pulmonar y un ingreso previo por insuficiencia cardiaca congestiva en otro centro hospitalario. El motivo del actual ingreso fue un nuevo cuadro de insuficiencia cardiaca biventricular con edema pulmonar e hipotensión asociada en relación con un episodio de fibrilación auricular rápida. Simultáneamente la paciente presentó una insuficiencia renal aguda prerrenal (creatinina 2,9 mg/dl) y una importante citolisis hepática (GOT 5480 U/L, GPT $5360 \mathrm{U} / \mathrm{L}$, bilirrubina $4,7 \mathrm{mg} / \mathrm{dl}$ ) con descenso asociado de la tasa de protrombina (Quick 33\%). En una ecocardiografía se observó una hipocinesia global del ventrículo izquierdo con una fracción de eyección del 39\% con dilatación del ventrículo derecho. La paciente fue tratada con digitálicos, diuréticos endovenosos y vasodilatadores periféricos con mejoría de los signos de fallo cardiaco y progresiva normalización tanto de la función renal como de la biología hepática.

Las figuras 1 y 2 muestran la evolución de las transaminasas y la tasa de protrombina en los 3 casos descritos.

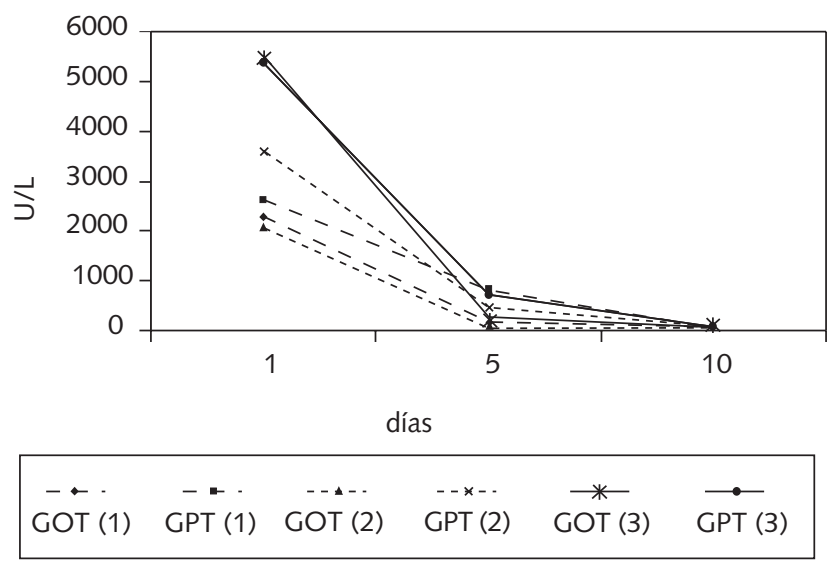

Fig. 1. Evolución de las transaminasas desde el momento del diagnóstico de la hepatitis isquémica en los casos 1, 2 y 3.

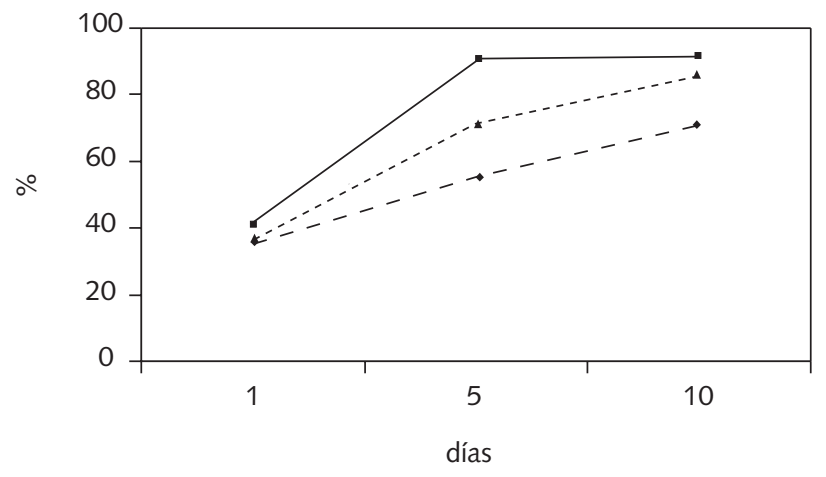

$-\bullet$ Caso 1

$\longrightarrow$ Caso 2

- - Caso 3

Fig. 2. Evolución de la tasa de protrombina.

\section{DISCUSIÓN}

La incidencia de la hepatitis isquémica no es bien conocida y depende del lugar donde se haya efectuado el estudio, aunque probablemente es baja. En una unidad coronaria la hepatitis isquémica se observó en el 2,6\% de todos los pacientes ingresados en un año y en el $21,9 \%$ de aquellos en los que se constató un bajo gasto cardiaco (4). En nuestro centro hemos observado 3 casos en un periodo de 1 año, que supone una incidencia del $2,7 \%$ entre todos los pacientes ingresados en el servicio de medicina interna con el diagnóstico de insuficiencia cardiaca.

Esta entidad se caracteriza por una elevación aguda y reversible de las transaminasas en el contexto clínico de cualquier proceso que condicione una reducción del flujo sanguíneo hepático, generalmente asociado a un bajo gasto cardiaco. Suele ser la responsable de aproximadamente el $50 \%$ de casos de citolisis hepática importante ingresados en centros hospitalarios (5). Siempre el médico está obligado a descartar otros procesos posiblemente responsables de una hepatitis aguda, especialmente las hepatitis virales y las farmacológicas. En esta situación la determinación de la enzima lactato deshidrogenasa (LDH) sérica puede ser de gran ayuda. Una elevación importante de las LDH con un cociente GPT/LDH inferior a 1,5 orienta claramente hacia una causa isquémica (6). A pesar de la importante alteración de la biología hepática, ésta suele resolverse con rapidez tras la mejoría de la causa subyacente sin dejar secuelas ni condicionar un peor pronóstico, el cual depende fundamentalmente de la enfermedad subyacente (7).

La etiopatogenia de la enfermedad no es bien conocida. Un factor determinante parece ser la hipotensión asociada a insuficiencia cardiaca izquierda que conduce a una reducción del flujo sanguíneo hepático (7), aunque éste no parece ser el único factor implicado. Seeto y cols. constataron que además de la hipotensión arterial es necesaria la existencia de insuficiencia cardiaca derecha, que condiciona una congestión venosa hepática, para que se desarrolle un cuadro de hepatitis isquémica (8). Ambos factores tienen una repercusión anatomopatológica bien diferenciada; la insuficiencia cardiaca derecha se traduce en una congestión pasiva crónica del parénquima hepático, mientras que la necrosis centrolobulillar es el resultado de una hipotensión arterial sistémica (9). En este sentido cabe destacar que no siempre se puede demostrar un episodio de hipotensión en todos los casos de hepatitis isquémica. Así, sólo en 2 de los 3 casos que presentamos se pudo constatar dicha hipotensión arterial, en cambio en los 3 existían signos de fallo cardiaco derecho.

En ocasiones una hipoxemia arterial grave sin insuficiencia cardiaca también puede causar una hepatitis hipóxica en pacientes con enfermedad pulmonar crónica (10), aunque el patrón hemodinámico es diferente del que se observa en los casos asociados a insuficiencia cardiaca. La hepatitis hipoxico-isquémica asociada a insuficiencia respiratoria grave es el resultado de una caída en las resistencias vasculares periféricas con un incremento del gasto cardiaco y una reducción del transporte tisular de oxígeno (11).

En conclusión, la hepatitis hipoxico-isquémica es infrecuente, pero no excepcional, en pacientes ingresados por fallo cardiaco. A pesar de la espectacularidad analítica del cuadro, su resolución es rápida y parece no tener ninguna repercusión sobre el pronóstico del paciente. 


\section{Bibliografía}

1. Gitlin N, Serio KM. Ischemic hepatitis: widening horizons. Am J Gastroenterol 1992; 87: 831-836.

2. Henrion J, Schapira M, Heller FR. Ischemic hepatitis: the need for precise criteria. J Clin Gastroenterol 1996; 23: 305.

3. Gibson PR, Dudley FJ. Ischemic hepatitis: clinical features, diagnosis and prognosis. Aus N Z J Med 1984; 14: 822-825.

4. Henrion J, Descamps O, Luwaert R, Schapira M, Parfonry A, Heller F. Hypoxic hepatitis in patients with cardiac failure: Incidence in a coronary care unit and measurement of hepatic blood flow. J Hepatol 1994; 21: 696-703.

5. Whitehead MW, Hawkes ND, Hainsworth I, Kingham JG. A prospective study of the causes of notably raised aspartate aminotrasnferase of liver origin. Gut 1999; 45: 129-133.

6. Cassidy WM, Reynolds TB. Serum lactic dehydrogenase in the differential diagnosis of acute hepatocellular injury. J Clin Gastroenterol 1994; 19: 118-121.
7. Fuchs S, Bogomolski-Yahalom V, Paltiel O, Ackerman Z. Ischemic hepatitis: clinical and laboratory observations of 34 patients. J Clin Gastroenterol 1998; 26: 183-186.

8. Seeto RK, Fenn B, Rockey DC. Ischemic hepatitis: clinical presentation and pathogenesis. Am J Med 2000; 109: 109-113.

9. Arcidi JM, Moore GW, Hutchins GM. Hepatic morphology in cardiac dysfunction: a clinicopathologic study of 1000 subjects at autopsy. Am J Pathol 1981; 104: 159-166.

10. Henrion J, Colin L, Schapira M, Heller FR. Hypoxic hepatitis caused by severe hypoxemia from obstructive sleep apnea. J Clin Gastroenterol 1997; 24: 245-249.

11. Henrion J, Minette P, Colin L, Schapira M, Delannoy A, Heller FR. Hypoxic hepatitis caused by acute exacerbation of chronic respiratory failure: a case-controlled, hemodynamic study of 17 consecutive cases. Hepatology 1999; 29: 427-433. 